\title{
Is tomorrow's car appealing today? Ethical issues and user attitudes beyond automation
}

\author{
Darja Vrščaj ${ }^{1}$ Sven Nyholm ${ }^{2} \cdot$ Geert P. J. Verbong ${ }^{1}$
}

Received: 14 March 2019 / Accepted: 11 December 2019 / Published online: 21 January 2020

(c) The Author(s) 2020

\begin{abstract}
The literature on ethics and user attitudes towards AVs discusses user concerns in relation to automation; however, we show that there are additional relevant issues at stake. To assess adolescents' attitudes regarding the 'car of the future' as presented by car manufacturers, we conducted two studies with over 400 participants altogether. We used a mixed methods approach in which we combined qualitative and quantitative methods. In the first study, our respondents appeared to be more concerned about other aspects of AVs than automation. Instead, their most commonly raised concerns were the extensive use of AI, recommender systems, and related issues of autonomy, invasiveness and personal privacy. The second study confirmed that several AV impacts were negatively perceived. The responses were, however, ambivalent. This confirms previous research on $\mathrm{AV}$ attitudes. On one hand, the AV features were perceived as useful, while on the other hand, their impacts were negatively assessed. We followed theoretical insights form futures studies and responsible research and innovation, which helped to identify that there are additional user concerns than what has been previously discussed in the literature on public attitudes and ethics of AVs, as well what has been envisioned by car manufactures.
\end{abstract}

Keywords Autonomous vehicles $\cdot$ Emerging technology $\cdot$ Prospective users $\cdot$ Recommender systems $\cdot$ User attitudes $\cdot$ Car manufacturer vision

\section{Introduction}

The 1982 science fiction movie Blade Runner opens with a police car flying over Los Angeles in what is supposed to be the year 2019. Later on, we learn that the car needs a driver. The interior of the Blade Runner cars is a small utilitarian space, much more like a military plane than the current car. The cars are box-shaped and their dashboards

Darja Vrščaj

d.vrscaj@tue.nl

Sven Nyholm

S.R.Nyholm@uu.nl

Geert P. J. Verbong

G.P.J.Verbong@tue.nl

1 School of Innovation Sciences, Eindhoven University of Technology, P. O. Box 513, 5600 MB Eindhoven, The Netherlands

2 Department of Philosophy and Religious Studies, Utrecht University, Janskerkhof 13, 3512 BL Utrecht, The Netherlands resemble 1990s computer screens, with a black background and fluorescent letters.

Although the cars envisioned by today's automobile industry cannot fly, they have some features that surpass the predictions in Blade Runner. Car drivers have been promised autonomous vehicles, enabling them to relax or work, while being driven. Several countries already allow autonomous cars on their roads, with some restrictions (Campbell 2017). Cars with more limited automation, for example Tesla's Model S with its autopilot feature, are already on the market. However, car manufacturers are now also envisioning cars as fully digitalized personal assistants, with Artificial Intelligence (AI) and Recommender Systems features, rather than utility machines transporting the user from A to B. Former visions might have gotten us used to the idea of flying cars and robots as personal assistants, but failed to prepare us for cars as intelligent personal assistants. Such previous visions also failed to prepare us for a spectrum of potential unwanted societal implications, e.g., new business models based on user data collection and user monitoring.

Furthermore, while the AI and Recommender Systems features play a dominant role in car manufacturers' visions 
about forthcoming AV developments, research about the potential negative implications of incorporating these features in AVs remains unexplored. These features have not been addressed in the literature discussing ethics and user attitudes of Autonomous Vehicles (AV). Rather, the focus has thus far been on studying the implications of the automated driving function, which is admittedly relevant and novel, but it is not the only feature to consider when discussing autonomous vehicles. In other words, by focusing on assessing the automated driving function, current research on future implications of the cars of the future is not discussing a broad enough spectrum of their potential implications.

The academic literature on the ethics and people's attitudes towards future cars has thus far focused on automation and safety concerns relating to potential accidents with automated cars (see, e.g., Kyriakidis et al. 2015; Liljamo et al. 2018; Molnar et al. 2018; Nyholm 2018a, b). Likewise, press coverage of the social and ethical aspects of cars of the future has also focused on these topics (e.g. Doctorow 2015; Marshall 2018; Wamsley 2018). A few studies on user attitudes have briefly mentioned privacy in relation to automation, e.g., user location, as one of the reasons users do not trust AVs, however, with conflicting evaluations regarding the significance of privacy concerns in relation to user trust (Kaur and Rampersad 2018; Kyriakidis et al. 2015; Liljamo et al. 2018). Furthermore, online commentaries have featured the issues of data collection and driverless cars' business models (Patel 2017; Ross 2018).

In this paper we aim to contribute to the research on attitudes and ethics of AVs, by studying user attitudes of AVs, beyond the automated driving function. We will focus on the attitudes of young people for two reasons. First, even though car developers refrain from providing a specific date for their ready-to-use AVs, this generation could be seen as its prospective users. ${ }^{1}$ Estimates about the market availability of AVs differ and are being updated with the advancing technological and political developments. Second, adolescents are expected to be more willing to accept AI technology compared to former generations, because they have been exposed to smart technology and AI since their early ages. In terms of the broader theoretical contribution, we aim to provide insights in how a societally desirable AVs would look like. For doing so, we focus on the future visions of today's car manufacturers, who present cars that are able to drive themselves, collect vast amounts of data about users, recommend and manage activities and places for users to visit. These visions are modelled on the success of the smart phone, and online website recommender systems. Also called Personal Digital Assistants, smart phones

\footnotetext{
$\overline{1}$ We gathered this information by interviewing car manufacturers at the IAA conference.
}

are multifunctional devices with various apps that owners can use for planning, entertainment, and socializing. Car manufacturers are increasingly following this example by imagining future cars as what might be called "smart phones on wheels." Just like smart phones, the envisioned cars will be equipped with a personal Recommender Systems ${ }^{2}$ (similar to the iPhone's Siri). These AI features will assist with basic cognitive tasks: searching, planning, messaging, and scheduling etc. (Danaher 2018), all while autonomously driving users to their desired destination.

These developments are partially driven by technological innovations relating to collecting and processing user data (IEEE 2018); car manufacturers are thus able to deploy new business models developed around the data generated by users (Templeton 2018). While making users' daily lives "easier" and fully utilizing the latest technological innovations, these developments also raise new social and ethical concerns. Such concerns about Recommender Systems (RS) and $\mathrm{AI}$ in general involve personal autonomy and privacy; the subordination of individuals' interests to those of large corporations; and moral responsibility regarding the value of human choice and agency (Danaher 2018; Floridi et al. 2018).

We argue that by focusing almost exclusively on automation, the current literature fails to fully consider the wider range of relevant car features and concerns - such as autonomy, invasiveness, dependency and personal privacy - that are central to the car industry's vision. For doing so we answer the following research questions: "what user attitudes and ethical issues have been identified in the literature about AVs?'; 'what attitudes do young adults have regarding the key features of the car of the future beyond automation?', "are the ethical concerns about AI and RS relevant in shaping young adults attitudes about AVs?'. Regarding the second question, we are particularly interested in whether the car industry's vision responds to potential users' attitudes and values.

Our paper starts with a literature review (Sect. 2), followed by theory and propositions (Sect. 3), methods (Sect. 4), two studies (Sect. 5), findings 1, 2 (Sects. 6, 7), discussion (Sect. 7) and conclusions (Sect. 8).

\section{Literature review}

In this section we explain the main concepts we are using in our paper, as well as review the literature on user attitudes and on ethics of automated cars.

\footnotetext{
2 The features that provide personalized recommendations about where to dine, plan your travel, pay or services, are called "recommender systems" (Lanzing 2018).
} 


\subsection{Autonomous vehicles}

Autonomous (also called self-driving, or driverless) vehicles sense their environment and navigate different traffic conditions with little or no human intervention for extended periods of time (Lin 2016; Maurer 2016; Skeete 2018). They do so by collecting real-time traffic data based on cameras and sensors, as well as by exchanging information with other vehicles and the infrastructure (Maurer 2016). This innovation is quickly advancing and is being tested on public roads and in public spaces. Nevertheless, it is uncertain how and when AVs will develop as there are unresolved technological, legal and ethical challenges ahead (Cohen and Cavoli 2018; Ryan 2019). Forecasts on market availability of AVs vary from hopeful estimations from the industry saying that AVs with little human assistance will be widely available in 3-5 years (Etherington 2017), to negative estimations saying that fully automated cars are unlikely to become a reality (Davies and Marshall 2019). Despite ongoing experimentation, AVs are an emerging technology, whose development and implications are still uncertain.

The vast majority of academic literature has focused on technological aspects of AVs; however, recently, there has been an increase of literature discussing societal implications of AVs (Cohen et al. 2018). The research on societal challenges has delved into the uncertainty regarding aspects of AVs relating to the use of AVs, wider impacts (e.g., on traffic flow, economy, land use and environment) governance structure, user acceptance and user attitudes, and ethical and legal challenges. Particularly public attitudes have been heavily researched, because the public has the potential to impact the technological development of AVs (Cavoli et al. 2017).

\subsection{User attitudes of autonomous vehicles}

The majority of user attitudes surveys have been focusing on investigating attitudes towards the automated driving feature. Specifically, they have assessed whether the public has positive or negative attitudes towards AVs. An extensive literature review conducted by Cavoli et al. (2017) confirms that there is uncertainty about the extent to which the public is interested in using or buying AVs. Therefore, despite several benefits of AVs that have been identified by industry experts and academics, e.g., increased safety, economic benefits, time saving for users, improved mobility for disabled and elderly people (Gurney 2016; Urmson 2015; Molnar et al. 2018), numerous surveys on user attitudes have continuously identified challenges in user trust and considerable resistance towards AV adoptance (Penmetsa et al. 2019) to the acceptance of autonomous cars (Molnar et al. 2018; Liljamo et al. 2018; Schoettle and Sivak 2014).
Several studies have identified that there is an increased likeliness that certain user profiles (men, young people, urban dwellers, technology enthusiasts, people living in certain geographical areas, e.g., California) could be interested in AVs (Cavoli et al. 2017). Public opinion is divided between, on one hand, the positive impacts: safety, perceived usefulness and perceived benefits (e.g., travel time or congestion reduction), and, on the other hand, negative implications relating to concerns about safety related to software malfunction and cybersecurity and costs, as the majority of surveyed participants would be reluctant to pay more for an AV compared to a non-automated car.

Recently, a few researchers have argued that a wider range of user concerns should be researched (Cohen et al. 2018; Taeihagh and Lim 2018). Cohen et al. (2018) have conducted a stakeholder workshop, which showed that stakeholders in the UK worry about additional aspects of AVs than those addressed in autonomous car innovation trajectories, e.g., cyber security, data ownership, sustainability, energy use and air quality, equity and access. We will further explore this; however, we surveyed a much larger group of participants in our studies.

\subsection{Ethics of autonomous vehicles}

AV researchers have started acknowledging that ethical concerns also play a role in the acceptance of emerging car technology, and should thus be linked to perspectives on user attitudes (see Adnan et al. 2018; Bonnefon et al. 2016; Frisoni et al. 2016). Zooming in on the ethical implications of future cars, ethics scholars have primarily been researching the automation aspect. They have identified two critical issues: firstly, which people at risk should be prioritized in unavoidable accident scenarios, the car occupants or other traffic participants; and secondly, how to allocate responsibility for accidents, in which people are harmed (see Hevelke and Nida-Rumelin 2015; Danaher 2018). ${ }^{3}$ Similarly, legal scholars have also dealt with issues of legal accountability (e.g., Beiker 2012; Gurney 2016; Marchant and Lindor 2012; Peterson 2012; Ravid 2014).

The ethics studies that have received the most attention outside academia are Bonnefon et al. (2016) and Awad et al. (2018), who studied people's attitudes to how automated cars should react in accident scenarios. Notably, one study on prospective user preferences relating to autonomous car crash scenarios revealed seemingly contradictory attitudes.

\footnotetext{
${ }^{3}$ Nyholm's (2018a) overview of the existing literature distinguishes three approaches: the so-called trolley problem (Bonnefon et al. 2015; Goodall 2016; Maurer et al. 2016; Wallach and Allen 2009), empirical ethics (Bonnefon et al. 2016; Kahane 2015), and traditional ethics theories, i.e. utilitarianism (Alfano 2013; Coeckelbergh 2016; Gogoll and Müller 2017; Goodall 2016; Keeling 2018; Leben 2017).
} 
While the study participants approved of other people using harm-minimizing cars that promote the greater good, they themselves did not want to buy or be forced to use such cars (Bonnefon et al. 2016). Furthermore, the same research group also mapped moral preferences in accident scenarios from laypeople in over 200 countries (Awad et al. 2018). These studies focus on crashes with automated cars—using what are called "trolley scenarios" (Nyholm and Smids 2016) after the famous philosophical trolley problem-to test people's attitudes.

\subsection{Recommender systems (RS) ethics}

Recommender systems (RS) provide personalized recommendations to a user based on a profile of the user's preferences and history, profiles of similar users, and/or sometimes on analysis of alternative recommended content (Choi et al. 2014). They are increasingly incorporated into cars. The recommender systems in mobile devices and vehicles differ from traditional online web recommendations by having enabled location-based recommendations (ibid).

Several ethical concerns relating to recommender systems have been identified in the academic literature. Several ethicists argue that outsourcing human tasks to RS, thereby letting automation creep into the mental and cognitive elements of tasks, is ethically contentious. Danaher has developed an overview identifying the following concerns: dehumanization (Frischmann 2014; Royakkers et al. 2018), cognitive degeneration (Carr 2015), and threats to personal freedom and autonomy (Crawford 2015; Floridi et al. 2018). Lanzing (2018), in turn, argues that recommender systems are ethically problematic, because they tend to hyper-nudge users without their knowledge (and based on commercial interests), thus undermining autonomy. Others raise questions such as whether RS threaten personal happiness and fulfilment. Krakauer (2016) and Morozov (2013) worry that RS and predictive analytics will impact our ability to make our own choices.

Furthermore, data collection is fuelling RS's machine learning as well as automatic decision-making (IEEE 2018). In recent years, we have witnessed controversies, where companies such as Facebook have used personal data for conducting experiments on users without their knowledge, or 'manipulated' data-driven personalized communication and behavioural targeting in the online realm (Lanzing 2018). Thus personal privacy is another topic of debate (see Borgesius et al. 2016; Floridi and Taddeo 2016; Lanzing 2018).

In the literature review above we concluded that while researchers have begun investigating people's attitudes and ethical concerns regarding AVs, they have focused on assessing the implications of automation, not of the AI and RS features. However, since AI and RS features are also increasingly becoming an integral part of AVs, they should also be assessed from a user perspective to identify whether they also pose concerns. We found that the more general literature on user concerns of AI and RS has identified a broader range of ethical concerns, such as personal privacy, invasiveness, dependency and autonomy, which may else be relevant to include in assessment of AVs.

\section{Theory}

With an increasing awareness of the potential impacts (often unwanted and unintended) of science and technology (e.g., privacy infringement) prospective analysis has become a prominent dimension of studying emerging technologies (Stilgoe et al. 2013). We follow the broader ideas of the Futures Studies field for studying the societal implications of the AVs emerging technology. The field rests on the premise that since the future is inherently uncertain, it should be explored by assessing numerous scenarios, which are then assessed in terms of plausibility as well as desirability (van Asselt 2010; Bell 2004). To account for future uncertainties, scenarios should not be mere extrapolations of the present to the future. Societally desirable scenarios are typically used to guide decisions in order to make informed decisions in the present, or to steer current events towards the preferable future (van Asselt 2010; Schwartz 1998).

Broadly speaking, there are two futures studies frameworks for studying emerging technologies: technology foresight and Technology Assessment/Responsible Research and Innovation. Their commonality is dealing with visions, which are defined as depictions of "a fuller portrait of an alternative world that includes revised social orders, governance structures, and societal values" (Konrad et al. 2017, p. 467). Whereas the former framework focuses on developing visions, the latter one goes further to assessing visions.

First, technology foresight is the process of systematically studying longer term futures of science and innovation with strategic goals (Hussain et al. 2017; van Lente 2012). Foresight is a successor of the technology forecasting approach. Forecasting extrapolates current trends into the future, which is seen as problematic, because it does not take complexity and uncertainty into account and because it assumes that the future can be predicted (Miles 2010). Technology foresight, on the other hand, accounts for the limitations of predicting the future while also considering the interaction between society and technology. Technology Foresight is commonly done through the approach of scenario planning, which are "a tool for ordering one's perceptions about alternative future environments in which one's decisions might be played out concretely, so people can help people make better decisions" (Schwartz 1998, p. 4; Ryan 2019). Scenarios, or 
visions, are typically developed through a combination of expert interviews and literature study (see Ryan 2019).

Second, once scenarios have been developed, they have to be assessed, which can be done through using the conceptual frameworks of Technology Assessment (TA)/Responsible Research and Innovation (RRI). The approaches are similar in exploring and advising decision makers about how to promote the development of technologies that would be aligned with societal values (Grunwald 2014). RRI aims to resolve the tension between the potential harms and benefits of emerging innovations through promoting ethically aligned technology designs, to increase their societal embedding (Stilgoe et al. 2013; von Schomberg 2011). We apply these frameworks, because they match our broader interest in the roles that prospective user attitudes play in the development of future cars. The framework is based on four interlinked and widely adopted RRI framework dimensions: anticipation, reflexivity, inclusion, and responsiveness. The anticipation, reflexivity, and inclusion dimensions are particularly relevant for our research aims. Anticipation relates to responding to uncertainty regarding potentially undesirable and unintended future impacts of emerging innovations. Reflexivity refers to scrutinizing the value systems framing particular innovations, which may not be universally held, as well as to avoiding "tunnel vision" by asking "what if questions". In turn, inclusion calls for assessing future visions through dialogues with direct and indirect stakeholders.

In the literature review section we have identified that the literature on user attitudes and ethics of AV has mainly looked at a limited vision of AVs. This gap was identified by looking at the visions put forward by the car manufacturers. Predominantly the focus in the literature has been on the automated driving, which is merely one of the envisioned $\mathrm{AV}$ features, as AI and RS are also increasingly implemented in cars. As such, future cars are studied as projections of current cars with a fully matured function for a full automated driving. The problem in these visions is that cars are still predominantly used as a means for getting from A to B, where their use is likely to change more to becoming personal assistants, or smart phones on wheels. Furthermore, these visions are not accounting for a prominent technology trend of convergence, where technologies that were previously unrelated, such as traditional cars and smart phones, become more closely integrated and unified. Such an example is Renault SYMBIOZ, which is an integrated house and an electric car that work together in harmony. ${ }^{4}$

Following the futures studies approach, in our study of user attitudes of AVs, we first developed likely future scenarios, in order to inform participants in the study about the envisioned car of the future. Following a common approach

\footnotetext{
$\overline{{ }^{4} \mathrm{https}: / / \mathrm{ww} w}$.youtube.com/watch?v=wWofSIB4osY.
}

for generating a vision (Ryan 2019), we wanted to interview to $\mathrm{AV}$ developers. We contacted the following companies: Amber Mobility, Audi, BMW, Citroen, Jaguar, MercedesBenz, Peugeot, Tesla, Volkswagen, and Waymo, but they did not grant us interviews. Hence, we turned to publicly available promotional materials (particularly videos). We examined the topics discussed at car industry conferences, for example recent conferences in Germany on functional safety and cyber security, ${ }^{5}$ and another on how to monetize car data. ${ }^{6}$ This helped to establish an idea of what the car industry believes users will desire, and what business models are envisioned for the car of the future, such as fully monetizing user data.

We made a 7.47 min video collage from well-known car brands' promotional materials. It featured 10 different car manufacturers, including BMW, Mercedes-Benz, Google car, Rolls Royce, Amber mobility, Volkswagen, Byton, Nissan, and Honda.

In selecting the video material, we aimed to capture presentations of numerous smart features beyond automation, e.g., recommender systems and mood detection. Furthermore, to trigger reactions to (assessment of) other features besides automation, we firstly showed automated driving and then proceeded to recommender systems used for controlling various aspects of users' daily lives. See the video using the following link: Car of the future video.

Before we proceed to presenting the study, we will explain the main three propositions we developed by integrating insights from the literature review and theory sections. The propositions explain what we expect to find in the surveys with prospective users.

Proposition $1(\mathrm{P} 1)$ The literature discussing user attitudes and ethics of AVs is incomplete, because it focuses on automation features. We propose that there are additional factors shaping young adults' attitudes, compared to what has been discussed in the literature. The following proposal further explains this assumption.

Proposition 2 (P2) Since RS and personal AI assistant features are increasingly becoming incorporated into $A V s$, they should be a focus of reflection on user attitudes towards AVs. Furthermore, we propose that the concerns discussed in the ethics literature on RS, such as personal privacy, invasiveness, dependency and autonomy, are also potentially worrisome to prospective users of AVs.

\footnotetext{
$\overline{5}$ https://www.euroforum.de/veranstaltung/pdf/p1107393en.pdf.

${ }^{6}$ https://veranstaltungen.handelsblatt.com/monetizing-cardata/confe rence-2019/.
} 
Proposition 3 (P3) The user attitudes literature postulates that users have ambivalent attitudes towards AVs, positive and negative. This led us to two sub-propositions, which build on each other.

$P 3$ a): user attitudes will be nuanced in a way, where they would find certain AV features attractive and certain unattractive, rather than being strictly supporting or objecting AVs.

$P 3$ b): prospective users might regard a specific AV feature as both useful, as well as worrisome.

\section{Methods}

We employed a mixed methods research approach, which involved collecting both qualitative data and quantitative, integrating the two forms of data (Creswell 2014). A mixed methods approach is based on the assumption that the combination of qualitative and quantitative approaches provides a more comprehensive understanding of a research problem than either approach alone (ibid.). We adopted the so-called "exploratory sequential mixed methods design", with which we started the qualitative phase (data collection and analysis) and then used the initial findings in the second quantitative phase (ibid.). The second data set was built based on the results of the initial data set to develop more specific measurements of user attitudes.

Following the mixed methods approach, we conducted two studies with bachelor students aged between 17 and 25 years. During the first round we collected 221 responses, and during the second one 271 responses. First, we researched what aspects of the car of the future prospective users are concerned about, besides automation, and why. Open-ended questions were necessary to meet the paper's aim was of avoiding to predetermine and influence respondents' answers. We wanted the respondents to, in their own words, describe why they find a car feature desirable/ undesirable. Open-ended questions ${ }^{7}$ have been said to be particularly fruitful when dealing with a novel field that is not yet structured and requires preliminary understanding (Patton 1990). We analysed the survey responses through a thematic analysis and category coding. This was done manually, since the surveys were also filled in manually. Furthermore, manual coding, as opposed to using a software, saved us time a lot of the answers were quite long and sometimes challenging to analyze. We followed a general approach of seeking connections within the data and generating initial

\footnotetext{
${ }^{7}$ See "Appendix" for survey questions.
}

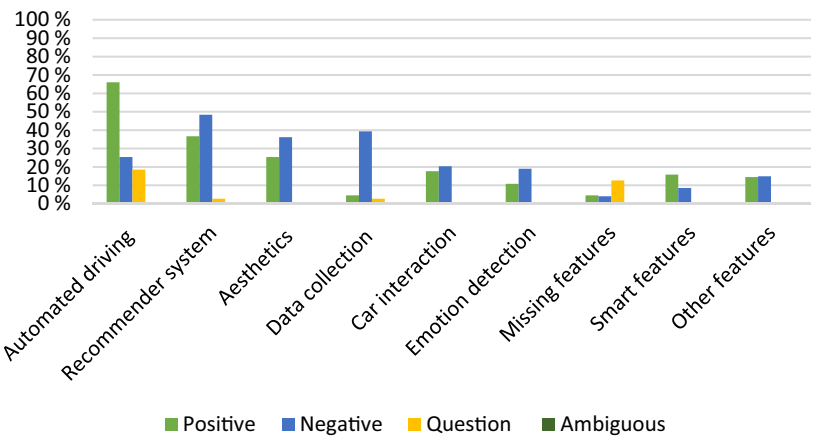

Fig. 1 Respondents' views on car features

codes, which led us to define thematic patterns, or themes (Williamson and Johanson 2018). Many of the themes in the survey responses overlapped with the features presented in the car of the future video, and we also noted additional themes, mostly relating to the potential impact of future cars. We identified three clusters of answers, ranging from technology features to societal implications: "car features and their impacts", "societal impacts", and "availability and accessibility". These subcategories are not mutually exclusive, and sometimes overlap. We transferred the data into Microsoft Excel, where we also calculated percentages and developed charts.

Second, we measured the intensity of the respondents' attitudes by conducting a second study using the Likert scale 1-7 (1 referring to "I do not agree at all", 7 referring to "I totally agree") for the different scales and items presented. We based the questions on nine of the most prominently answered responses gathered in the previous survey round. We analysed the results with the SPSS Statistics software for Windows, download version 25 , using the descriptive statistics function. This enabled us to calculate the means and standard deviations for each of the nine questions. We downloaded the results in charts, which we will present below.

\section{Study I results}

In this section we will introduce the findings of the qualitative study in three subsections: car features, social impacts and availability and accessibility.

\subsection{Car features (and their impacts) (Fig. 1)}

Automated driving:

- Positive (60.1\%) Car contributes to safety ("eliminating human error"), car pick-up; the ease of driving, driving assistance, "ability to still drive sometimes", or "take over control". 
- Negative (23\%) Lacking control, "not having the option to disable (not use) certain features", otherwise the study participants "would not feel safe", nor "trust the car".

- Question (16.9\%) The lack of information about safety in the video; questioned the vehicle's safety, as well as the user's role.

$\mathrm{AI}$ and recommender systems:

- Positive (41.5\%) "Hana seems really cool", "understands how you think", offering "some level of assistance", "following your needs" while "adapting to the user". Personalized journey or navigation recommendations ("smarter routes", "predicting what happens on the road") received $35.8 \%$ of the total positive responses $41.5 \%$.

- Negative (54.9\%) Lack of control and freedom, the car having "too much autonomy", being "too dominant" or "too intrusive in people's daily lives"; disapproving the car becoming driver's "assistant", "psychologist", or "friend". the personalized recommendations ("the car interfering in their personal life") spooky", "too invasive"; "personal recommendations would lead to consumerism".

- Question (3.1\%).

- Ambiguous (0.5\%).

Aesthetics:

- Positive (40.6\%) Sleek, modern aesthetics envisioned by the car manufacturers, comfortable interiors received a few positive responses.

- Negative (58\%) "Modern", "weird", and "unnecessarily fancy" designs of the cars of the future.

- Ambiguous (1.4\%).

Data collection:

- Positive (9.7\%) Merging different types of personal data with the car, like agenda and phone information.

- Negative (84.5\%) Personal privacy, e.g., "AI knows your mental and physical state, I want privacy"; personal data (e.g., bank account and contacts) stored in the car, the "robot knows everything about you - what if this info is shared with the wrong people - Facebook already knows too much"; merging user information, daily life, or agenda with the car, the car is constantly collecting and analysing data.

- Ambiguous (5.8\%).

Car interaction:

- Positive (46.4\%) Hand, finger, gesture tracking, or control functions.
- Negative (53.6\%) "Confusing", "too distracting"; voice control as "too invasive", "annoying", "creepy", e.g., "sometimes in the morning you just want to be left alone instead of talking to Hana".

Emotion and mood detection:

- Positive (34.8\%) Personalized recommendations relating to the journey and navigation; beneficial safety impact, particularly the fatigue detection feature. car adaptation to one's mood and its assistance with reducing stress levels.

- Negative (60.9\%) Recommendations relating to "personal life" are "creepy" and "invasive", "I do not see the point of AI scanning my emotions. This invasion of privacy seems like a huge waste of money and effort, if all it can do is talk to me in a condescending voice and pick the easiest route, something every navigation system can already do without having to scan my face".

Missing features:

- Missing features (59.6\%) Seatbelts, a coffeemaker, cooking facilities, cup holders, and more futuristic features as seen in sci-fi films like Blade Runner, namely, flying cars.

- Positive (21.3\%).

- Negative (19.1\%).

Smart features ("rotating seats", "doors opening automatically", "folding car parts" like arm-rests):

- Positive (63.6\%) They adapt to the driver, are "modern".

- Negative (34.6\%) "Overload of functions".

Other features:

- Positive (49.2\%) Operating the car with a smart phone or smart watch "in case you forget your keys": the functions that enable the car to order and pay online for a product like coffee; unspecified smart technology; and omnipresent connectivity.

- Negative (50.8\%) Operating the car with a smart phone or smart watch "could lead to a cyber-attack" or "the phone could drain the car battery".

The automated driving feature was the most discussed feature as well as the most positively perceived AV feature. In contrast, the AI and RS feature was the second most discussed feature and also the most negatively assessed feature. We identified several additional concerns regarding $\mathrm{AVs}$, including data collection and emotion and mood detection. 


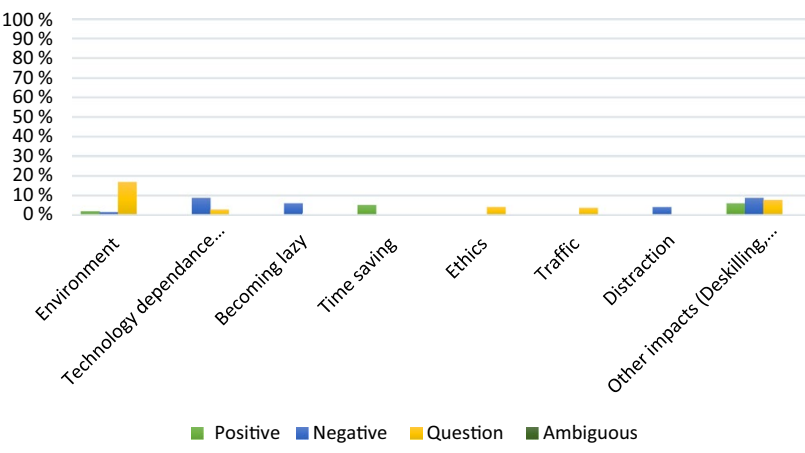

Fig. 2 Respondents' views on the societal impacts of future cars

Numerous features were assessed more impartially, such as aesthetics, car interaction and smart features.

\subsection{Societal impacts (Fig. 2)}

\section{Environment:}

- Positive (9.1\%) The cars of the future running on sustainable fuel.

- Negative (6.8\%) The lack of attention to environmental issues, e.g., "it irritates me that instead of solving real problems like global warming, we are trying to make life easier in the most futile way".

- Question (84.1\%) Fuel use, e.g., are the cars electric, something the video did not mention.

Technology dependence:

- Negative (76\%) "Overdependence on cars to assist you in everyday life, or mentally", "humans seem unable to take care of themselves", "technology failure", "someone could hack into the car", "autonomous cars make the wrong decision", "lack of information on risks if technology fails".

- Question (24\%) "What about safety - as in cyber security?"

Becoming lazy:

- Negative (81.25\%) The impact of future cars on people's sense of adventure, making people "boring", "lazy", "docile", or "self-helpless". "I do not understand why this is called progress. I don't want to become a useless human unable to do anything"; "human activity is reduced to a minimum because of AI, we don't even have to think about our commitments or desires, because AI takes care of those for you".

- Question (12.5\%).

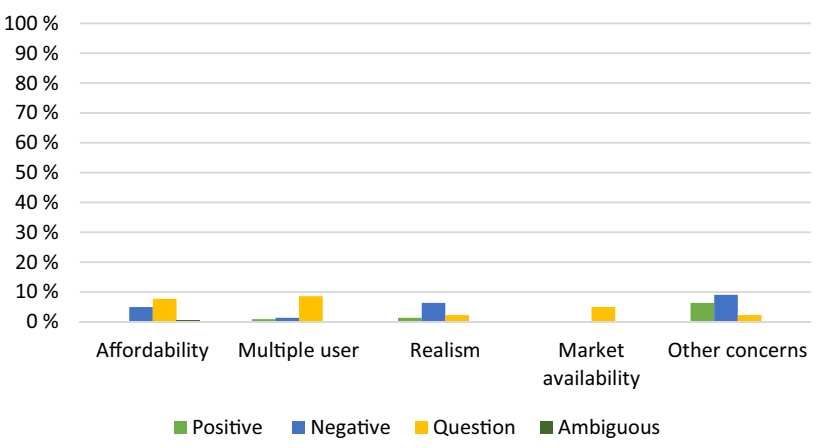

Fig. 3 Respondents' concerns about the car of the future

Saving time:

- Positive (84.6\%) "Less focus on driving"; enables the driver to be faster and more efficient; offers "a more productive drive", because you are "able to work while driving", and "read a book or talk to a friend".

- Question (15.4\%) Would the car save time, or would the time saved be spend productively.

Ethics:

- Negative (18.2\%) the car lacks ethics, e.g., "the car decides what is ethical".

- Question (81.8\%) "Who will go to jail if the car kills people?".

Distraction:

- Negative (100\%) Distract the user during the drive, e.g., the car has too many features and provides too much stimulation for the driver (the entertainment system, big screens, and the voice operation option).

Traffic:

- Question (100\%) Cars' implementation and function in traffic, "would the roads change if cars change?", "how would they interact with other cars", "what would be the impact on congestion?", "will we still need new traffic signs?", and "how would it work in India's traffic?"

Other societal impacts:

- Positive (26.5\%) Saving time.

- Negative (38.8\%) That cars would "make driving boring", and users would "miss the fun and pleasure of driving"; social alienation and isolation, "less real interaction with other people", de-skilling, including concerns about humans' capabilities becoming less sophisticated, "los- 
Table 1 Means and standard deviation (SD) of the Study II

\begin{tabular}{lrr}
\hline Question (Likert scale 1-7) & Mean & SD \\
\hline How useful, or helpful, do you find the AI personal assistant in the car of the future (e.g., Hanna)? & 4.37 & 1.581 \\
How invasive, or creepy, do you find the AI personal assistant in the car of the future in your private life? & 5.33 & 1.577 \\
How controlling (jeopardizing your autonomy) do you find the car the AI personal assistant in the car of the future? & 4.96 & 1.551 \\
How worried are you that the future car will compromise your personal data? & 4.98 & 1.664 \\
How worried are you that the future car will merge your information from different devices and online platforms? & 4.87 & 3.86 \\
How helpful do you find the car's emotion and mood detection function (e.g., sleepiness, stress)? & 1.737 \\
How invasive or creepy do you find the car's emotion and mood detection (e.g., sleepiness, stress)? & 1.739 \\
How worried are you about depending on the car, e.g., to assist you in your everyday life, or with your mental tasks? & 4.22 & 1.734 \\
How worried are you about the car of the future making people boring or lazy? & 4.867 & 1.93 \\
\hline
\end{tabular}

ing driving occupations"; "missing human aspect" or "human side", "humanity - the devices are replacements of our own minds", "there is no human contact".

- Question (34.7\%).

We identified several user concern regarding societal implications potentially posed by AVs, including environmental concerns, technology dependence, becoming docile, being distracted. The only predominantly positively assessed implication was time saving.

\subsection{Availability and accessibility (Fig. 3)}

Affordability:

- Question (58.6\%) A high and unaffordable price for the car of the future, "Who will use it? Only the rich?"; exclusivity, that the envisioned cars are merely "for a small group of people, high society", "how will it operate within different classes?" A couple of responses to the survey question 'is there anything missing?' mentioned lacking "enough money to buy one".

- Negative (38\%).

- Ambiguous (3.4\%).

Multiple users:

- Positive (8.3\%) Cars could be shared by multiple drivers, even though the video did not feature car-sharing.

- Negative (12.5\%) "Child-unfriendly" or "grandfatherunfriendly".

- Question (79.2\%) Whether multiple people such as family members can use the car.

Realism:

- Positive (13.6\%) Cars are a good solution for an ongoing challenge.
- Negative (63.6\%) The lack of reality in the car manufacturers' vision, "it's too futuristic", "humanity does not work like this, people are not ready to rely on and entrust their lives to a robotic system", there is a "big discrepancy with current cars", "the AI only works with futuristic and luxurious cars".

- Question (22.8\%) "Missing an actual solution to a real problem".

Market availability:

- Question (100\%) "How far on is the development?", "when will these cars be available?".

Other concerns:

- Positive (35.9\%) Positive reactions regarding increased mobility for disabled, blind, and elderly people.

- Negative $\mathbf{( 5 1 . 3 \% )}$ ) The car is a product for men and that there is a distinct "lack of women drivers", "missing women and people who are not white men", and "women only in AI or servant voices"; the cars are "too luxurious", "I don't like their initial function, just luxury".

- Question (12.8\%) Similar negative concerns expressed as questions.

We identified numerous concerns regarding accessibility and availability of AVs. Amongst most prominently mentioned concerns were affordability, market availability and the possibility of sharing the car with several users.

\section{Study II}

Based on the results of the first survey round, we ran another survey, in which we quantitatively researched the intensity of the prospective user attitudes towards the AI personal assistant function identified in the first study. The study respondents $(n=251)$ perceived the assistant and mood detection 
features as helpful. The participants were between 17 and 25 years old, with an average age of 19.70 . User attitudes were nuanced and ranged from both positive to negative. However, to a smaller degree, we identified more negative and worried attitudes than positive ones (Table 1).

\subsection{Attitudes about the car Al and RS (personal assistant) features}

The AI and RS features were perceived as helpful and useful $(M=4.37$; Std. Dev.: 1.581). However, their implications were at the same time, with an even stronger opinion, perceived negatively. Specifically, we tested the implications that relate to this feature being invasive and creepy $(M=5.33$; Std. Dev.: 1.577); as well as controlling in the sense of jeopardizing one's autonomy $(M=4.96$; Std. Dev.: $1.551)$.

\subsection{Attitudes towards car emotion and mood detection features}

Similarly to the results shown in the previous section, we found that the participants assessed the cars' emotion and mood detection features ambivalently. On one hand, the car emotion and mood detection features were perceived as useful or helpful ( $M=3.86$; Std. Dev.: 1.739), although to a smaller extent than the above-mentioned AI personal assistant. On the other hand, this feature was also perceived as invasive or creepy, and on a much higher scale than it was perceived to be helpful ( $M=5.08$; Std. Dev.: 1.734).

\subsection{Concerns about the car of the future}

Several features of the car of the future were assessed to be worrisome: The highest level of concerns was found in relation to the cars' compromising respondents' personal data $(M=4.98$; Std. Dev.: 1.664). Also relating to the issues of data that the car of the future will be collecting, the respondents expressed concerns about the cars merging their information from different devices and online platforms $(M=4.87$; Std. Dev.: 1.737). We tested how concerned prospective users are about two additional features: for inducing dependence on the car, e.g., to assist in everyday life, or with mental tasks ( $M=4.22$; Std. Dev.: 1867$)$; and about making people boring or lazy $(M=4.69$; Std. Dev.: 1.93$)$.

\section{Discussion}

In this section we will discuss whether our findings confirmed, or contradicted, our propositions.

The first proposition was supported, as our research showed that there are more user concerns at stake than those discussed in the literature on ethics and public attitudes on AVs. The literature discusses the concerns relating safety, reduced travel time, cyberattacks, informational privacy, e.g., data ownership, accident responsibility, energy use and access (Cavoli et al. 2017; Cohen et al. 2018; Liljamo et al. 2018; Molnar et al. 2018; Nyholm and Smids 2016). On one hand, our Study I confirmed that all of the concerns researched thus far are relevant factors in shaping attitudes of young adults regarding AVs. However, on the other hand, the study also confirmed that there are additional issues that have not been previously discussed extensively in the literature. We have identified that the following user concerns and AV features should be included in future discussions about AVs: autonomy and control over the technology and personal data, personal privacy, modern sleek AV design, technology dependence, becoming lazy or docile, de-skilling, lack of social interaction, car-and ride sharing possibility. This is not to say that the literature is not completely wrong, but rather that it is limited, because the AV industry is envisioning various additional developments.

The RRI conceptual framework is instrumental in understanding why certain user concerns may have been omitted from academic discussions. Anticipation, a crucial dimension of responsible governance of emerging technology, is challenged by projecting current images of the car as a device taking us from $\mathrm{A}$ to $\mathrm{B}$, rather than anticipating the impact of new emergent uses, such as cars as personal assistants governing our daily lives or "smartphones on wheels". Consequently, several above-mentioned potential implications, and uses of autonomous cars, have not been anticipated in the literature (Ryan 2019).

The second proposition was also confirmed as the AI and RS features were the second most often addressed AV feature (the automated driving feature was addressed the most). Furthermore, AI and RS were the features that were the most negatively evaluated. In other words, the participants in our studies were nearly as concerned with aspects related to AI and RS as they were with automation. The concerns identified in Study I include: autonomy (AV has too much control in planning ones daily life, e.g., through functioning as a personal assistant), invasiveness ("the car is too involved in your personal life"), and personal privacy (the car is collecting personal information such as users mental state, bank account number, contacts, agenda,...). To a smaller extent the issue of making humans docile was addressed. In Study II we identified that while the AI personal assistant feature was perceived as massively invasive and creepy $(M=5.33)$, and jeopardizing autonomy $(M=4.96)$, it was also perceived as a helpful and a useful function $(M=4.37)$. To sum up, this study shows that several issues, and uses of AVs, that have been identified in the ethics literature researching the implications of AI and RS (but not in AV literature) are relevant in shaping user attitudes about AVs. 
The proposition P3a was supported; Study II confirmed the findings about user attitudes regarding AVs being ambivalent, which has also been identified in previous research on user attitudes of AVs (Cavoli et al. 2017). User attitudes are nuanced. Nearly every AV feature was perceived positively by roughly half of the participants, but also negatively by the other half. Nevertheless, there were some exceptions, such the AV data collection feature, which was mainly assessed negatively $(84.5 \%)$. On the flip side, the automated driving feature was largely positively evaluated $(60.1 \%$, compared to $23 \%$ of negative $16.9 \%$ having additional questions). As most of the other AV features were assessed more neutrally (having gathered nearly the same amount of positive and negative responses), the overall young respondents' attitudes towards AVs remain ambiguous. However, we identified that for a small percentage, the negative attitudes towards AVs prevailed.

The subproposition building on the previous proposition, namely, P3b, was roughly supported and offered another perspective in understanding the ambivalences in user attitudes. On one hand, we identified that prospective users are finding car features both useful and concerning. First, while the AI personal assistant was perceived as having numerous issues such as being massively invasive and creepy $(M=5.33)$, as well as jeopardizing autonomy $(M=4.96)$, it was also perceived as helpful and useful $(M=4.37)$. The attitudes about the emotion and mood detection function were also perceived ambivalently, albeit as less helpful and useful $(M=3.86)$ while significantly invasive or creepy $(M=5.08)$. Furthermore, the majority of the study participants consistently reported being worried that the car will compromise their personal data $(M=4.98)$, and merge their data $(M=4.87)$, make them docile (4.69) and dependent $(M=4.22)$. Therefore, while AVs, as currently envisioned by the car manufacturers, are posing several concerns, this technology is not perceived as a strictly negative, or an undesirable, mobility solution. However, on the other hand, while the study results clearly show that young adults have ambivalent attitudes towards AV features (P3a) and that they find them both concerning and useful (P3b), it is still possible to identify, even if by a small percentage, whether a certain AV feature was perceived more positively of more negatively.

Despite the considerable amount of negative user attitudes and the user concerns identified during our studies, one should not simply conclude that prospective users will not be interested in using AVs. Several new technologies such as smart phones and social media have shown that sometimes people are willing to tolerate the negative impacts of a technology for the sake of enjoying the useful and helpful side of the technology. Looking into the future in which the AVs will be more widely used, people may prefer using the technology over not using it. Prospective users, therefore, might accept, e.g., handing over the control over their personal data, because they will not want to miss out on the benefits of being taken around in a personalized assistant on wheels.

\section{Conclusion}

In terms of our broader theoretical contribution, we have aimed to provide insights into what a societally desirable AV would look like. Our study suggests that it is challenging to develop such an image as there are more issues at stake than have been previously acknowledged by the stakeholders. On one hand, the car manufacturers' promotional material envisioning future $\mathrm{AVs}$ as digital personal assistants, which increasingly govern various aspects of users' daily lives, is not in line with the values of prospective users and the ethical restrictions suggested by AI ethicists. Thus, $\mathrm{AI}$ and RS, which are increasingly being embedded in various technologies, conflict with some of the values widely held in society today. ${ }^{8}$ Accordingly, the additional public concerns should be addressed and included in visions on $\mathrm{AVs}$ to stimulate the development of desirable future $\mathrm{AVs}$ and greater acceptance of such AI-intensive technologies.

The often-mentioned example of the smart phone becoming a widely used innovation, despite prospective users' initial negative evaluations, highlights two points. One, the challenging nature of anticipating future user attitudes, due to our ignorance regarding the future (technologies may be used in unexpected ways, and user values are likely to coevolve through the use of new technology, Jasanoff 2004). Two, the pertinence of seeking inclusive anticipation, since technologies tend to pose unintended and sometimes undesirable impacts, as in the case of the smart phone, concerning privacy, exploitation of scarce materials, social life dynamics, and so on. Yet, since the smart phone has become so embedded in our daily lives, it is now difficult to change it to avoid some of its negative impacts-a prime example of the Collingridge (1980) dilemma.

Seeking alignment early on in the innovation process is thus both necessary and challenging. We present our study as an example of inclusive anticipation. Instead of asking for views on predetermined issues, we used a vision developed by car manufacturers and invited prospective users to share their thoughts regarding any aspect of the car of the future. This interaction with users revealed the scope for further research on how the values held by car designers and other tech companies could be negotiated (Van Der Hoven 2013) to achieve better alignment of future AVs with prospective users' attitudes. For example, the survey participants on one hand perceived the AI personal assistant in the car of the future as useful, while, on the other hand, also as invasive

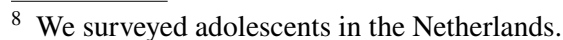


and jeopardizing their autonomy. Further research should explore (and consult users about) which mechanisms and AI principles would need to be put in place in order for users to feel that their values are not jeopardized, that they are in control, and that cars are not invading their personal sphere. New business models and technology designs could facilitate disabling certain options, limit the car data collection, and provide full transparency on how the stored data could be used, perhaps even consulting the users before selling the data to third parties. We thus propose sometimes compromising, or giving up part of the technological sophistication, for protection of the key user values, such as, autonomy.

The main limitation of our study relates to the difficulties of identifying of emerging user attitudes. As an emerging technology, AVs will evolve in a dynamic process with an uncertain outcome. This can be seen as the above described process of co-creation (Jasanoff 2004), according to which user attitudes will be co-created in relation with how the car manufactures will develop AVs.

In conclusion, although the flying cars seen in Blade Runner are not yet on the horizon, the emerging smartphones on wheels are posing new ethical concerns that require further research. This research should address the values at the heart of prospective users' worries to explain why our respondents feared cars being able to detect their emotions and gather personal information.

Acknowledgements We would like to thank the Netherlands Ministry for Infrastructure and Water Management for co-funding this research project. We also thank all the survey participants and the anonymous reviewers.

Open Access This article is licensed under a Creative Commons Attribution 4.0 International License, which permits use, sharing, adaptation, distribution and reproduction in any medium or format, as long as you give appropriate credit to the original author(s) and the source, provide a link to the Creative Commons licence, and indicate if changes were made. The images or other third party material in this article are included in the article's Creative Commons licence, unless indicated otherwise in a credit line to the material. If material is not included in the article's Creative Commons licence and your intended use is not permitted by statutory regulation or exceeds the permitted use, you will need to obtain permission directly from the copyright holder. To view a copy of this licence, visit http://creativecommons.org/licenses/by/4.0/.

\section{Appendix}

\section{Study I}

Which features of the cars in the video do you find attractive?

Is there anything you do not like?

Do you think anything is missing?

Study II
On a scale of 1-7 how useful, or helpful, do you find the AI personal assistant in the car of the future (e.g., Hanna)?

On a scale of 1-7 how invasive, or creepy, do you find the AI personal assistant in the car of the future in your private life?

On a scale from 1 to 7 how controlling (jeopardizing your autonomy) do you find the car the AI personal assistant in the car of the future?

On a scale of 1-7 how worried are you that the future car will compromise your personal data?

On a scale of 1-7 how worried are you that the future car will merge your information from different devices and online platforms?

On a scale of 1-7 how helpful do you find the car's emotion and mood detection function (e.g., sleepiness, stress)?

On a scale of 1-7 how invasive or creepy do you find the car's emotion and mood detection (e.g., sleepiness, stress)?

On a scale of 1-7 how worried are you about depending on the car, e.g., to assist you in your everyday life, or with your mental tasks?

On a scale of 1-7 how worried are you about the car of the future making people boring or lazy?

\section{References}

Adnan N, Nordin SM, Bahruddin MAB, Ali M (2018) How trust can drive forward the user acceptance to the technology? In-vehicle technology for autonomous vehicle. Transport Res Part A Pol Pract 118:819-836. https://doi.org/10.1016/J.TRA.2018.10.019

Alfano M (2013) Character as moral. Fiction. https://doi.org/10.1017/ CBO9781139208536

Awad E, Dsouza S, Kim R, Schulz J, Henrich J, Shariff A, Rahwan I (2018) The moral machine experiment. Nature 563(7729):59-64. https://doi.org/10.1038/s41586-018-0637-6

Beiker S (2012) Legal aspects of autonomous driving. Santa Clara Law Rev 52:1145-1156

Bell W (2004) Foundations of futures studies, vol 1. Human Science for a New Era. http://books.google.dk/books?id=ILJ_pfMgLqsC

Bonnefon J-F, Shariff A, Rahwan I (2015) The social dilemma of autonomous vehicles. Science. https://doi.org/10.1126/scien ce.aaf 2654

Bonnefon J-F, Shariff A, Rahwan I (2016) The social dilemma of autonomous vehicles. Science (New York) 352(6293):1573-1576. https://doi.org/10.1126/science.aaf2654

Borgesius FJZ, Trilling D, Möller J, De Vreese CH, Helberger N (2016) Should we worry about filter bubbles? Internet Pol Rev 5(1):1-16. https://doi.org/10.14763/2016.1.401

Campbell P (2017) Governments compete to take the wheel on rules for self-driving cars. Financial Times. https://www.ft.com/conte nt/ff19d296-af44-11e7-8076-0a4bdda92ca2. Retrieved 5 Feb 2019

Carr N (2015) The glass cage: where automation is taking us. The Bodly Head, London. ISBN 978-1-84792-308-0. https://www. worldcat.org/title/glass-cage-where-automation-is-taking-us/ oclc/905444467?referer $=$ di\&ht=edition

Cavoli C, Phillips B, Cohen T, Jones P (2017) Social and behavioural questions associated with automated vehicles: a literature review. UCL Transport Institute, London

Choi J, Lee HJ, Sajjad F, Lee H (2014) The influence of national culture on the attitude towards mobile recommender systems. Technol Forecast Soc Change 86:65-79. https://doi.org/10.1016/j.techf ore.2013.08.012 
Coeckelbergh M (2016) Responsibility and the moral phenomenology of using self-driving cars. Appl Artif Intell 30(8):748-757. https ://doi.org/10.1080/08839514.2016.1229759

Cohen T, Cavoli C (2018) Automated vehicles: exploring possible consequences of government (non)intervention for congestion and accessibility. Transp Rev 39(1):129-151. https://doi. org/10.1080/01441647.2018.1524401

Cohen T, Stilgoe J, Cavoli C (2018) Reframing the governance of automotive automation: insights from UK stakeholder workshops. J Responsible Innov. https://doi.org/10.1080/23299460.2018.14950 30

Collingridge D (1980) The societal control of technology. Pinter, London. https://openlibrary.org/books/OL14443859M/The_socia 1_control_of_technology

Crawford MB (2015) The world beyond your head: on becoming an individual in an age of distraction. Farrar, Straus and Giroux, New York

Creswell JW (2014) Research design: qualitative, quantitative and mixed methods approaches. Sage, Los Angeles

Danaher J (2018) Towards an ethics of AI assistants: an initial framework. Philos Technol. https://doi.org/10.1007/s1334 7-018-0317-3

Davies A, Marshall A (2019) Are we there yet? A reality check on self-driving cars/WIRED. https://www.wired.com/story/futur e-of-transportation-self-driving-cars-reality-check/\#. Accessed 3 Dec 2019

Doctorow C (2015) The problem with self-driving cars: who controls the code? Technology. The guardian. https://www.theguardia n.com/technology/2015/dec/23/the-problem-with-self-drivingcars-who-controls-the-code. Accessed 5 Feb 2019

Etherington D (2017) BMW's self-driving car will aim for full level 5 autonomy by 2021ITechCrunch. https://techcrunch .com/2017/03/16/bmws-self-driving-car-will-aim-for-fulllevel-5-autonomy-by-2021/?guccounter $=1$ \&guce_referrer us $=$ aHR0cHM6Ly 93d3cuZ29vZ2x1LmNvbS 8\&guce_refer rer_cs=pmZoo_6QvPsHoyCNnb8SnQ. Accessed 30 Oct 2019

Floridi L, Taddeo M (2016) What is data ethics? Philos Trans A Math phys Eng Sci. https://doi.org/10.1098/rsta.2016.0360

Floridi L, Cowls J, Beltrametti M, Chatila R, Chazerand P, Dignum $\mathrm{V}$ et al (2018) AI4People-an ethical framework for a good AI society: opportunities, risks, principles, and recommendations. Mind Mach 28(4):689-707. https://doi.org/10.1007/s1102 3-018-9482-5

Frischmann BM (2014) Human-focused turing tests: a framework for judging nudging and techno-social engineering of human beings. SSRN Electron J. https://doi.org/10.2139/ssrn.2499760

Frisoni R, Dall'Oglio A, Nelson C, Long J, Vollath C, Ranghetti D, McMinimy S (2016) Research for TRAN committee-selfpiloted cars: the future of road transport? EU directorate-general for internal policies policy department b: structural and cohesion policies transport and tourism. http://www.europarl.europ a.eu/RegData/etudes/STUD/2016/573434/IPOL_STU\%28201 6\%29573434_EN.pdf. ISBN 978-92-823-9055-9

Gogoll J, Müller JF (2017) Autonomous cars: in favor of a mandatory ethics setting. Sci Eng Ethics 23(3):681-700. https://doi. org/10.1007/s11948-016-9806-x

Goodall NJ (2016) Away from trolley problems and toward risk management. Appl Artif Intell 30(8):810-821. https://doi. org/10.1080/08839514.2016.1229922

Grunwald A (2014) Technology assessment for responsible innovation. Responsible Innov 1 Innov Sol Glob Issues. https://doi. org/10.1007/978-94-017-8956-1_2

Gurney JK (2016) Crashing into the unknown: an examination of crash-optimization algorithms through the two lanes of ethics and law. Albany Law Rev 79(1):183-267
Hevelke A, Nida-Rümelin J (2015) Responsibility for crashes of autonomous vehicles: an ethical analysis. Sci Eng Ethics 21(3):619-630. https://doi.org/10.1007/s11948-014-9565-5

Hussain M, Tapinos E, Knight L (2017) Scenario-driven roadmapping for technology foresight. Technol Forecast Soc Chang 124:160-177. https://doi.org/10.1016/j.techfore.2017.05.005

IEEE (2018) Ethically aligned design-version II overview. IEEE Control Syst Mag. https://doi.org/10.1109/MCS.2018.2810458

Jasanoff S (2004) States of knowledge: the co-production of science and the social order. In: States of knowledge. https://doi. org/10.4324/9780203413845

Kahane G (2015) Sidetracked by trolleys: why sacrificial moral dilemmas tell us little (or nothing) about utilitarian judgment. Soc Neurosci 10(5):551-560. https://doi.org/10.1080/17470 919.2015.1023400

Kaur K, Rampersad G (2018) Trust in driverless cars: investigating key factors influencing the adoption of driverless cars. J Eng Tech Manage 48:87-96. https://doi.org/10.1016/J.JENGT ECMAN.2018.04.006

Keeling G (2018) Legal necessity, pareto efficiency and justified killing in autonomous vehicle collisions. Ethic Theory Moral Pract 21(2):413-427. https://doi.org/10.1007/s10677-018-9887-5

Konrad K, van Lente H, Grovers C, Selin C (2017) Performing and governing the future in science and technology. In: Felt U, Fouché R, Miller CA, Smith-Doerr L (eds) The handbook of science and technology studies. 4th edn. The MIT Press, Cambridge, Massachusetts, pp 465-493

Krakauer D (2016) Will A.I. harm us? Better to ask how we'll reckon with our hybrid nature. http://nautil.us/blog/will-ai-harm-us-bette r-to-ask-how-well-reckon-with-our-hybrid-nature. Retrieved $5 \mathrm{Feb}$ 2019

Kyriakidis M, Happee R, de Winter JCF (2015) Public opinion on automated driving: results of an international questionnaire among 5000 respondents. Transport Res Part F Traffic Psychol Behav 32:127-140. https://doi.org/10.1016/J.TRF.2015.04.014

Lanzing M (2018) "Strongly recommended" revisiting decisional privacy to judge hypernudging in self-tracking technologies. Philos Technol. https://doi.org/10.1007/s13347-018-0316-4

Leben D (2017) A Rawlsian algorithm for autonomous vehicles. Ethics Inf Technol 19(2):107-115. https://doi.org/10.1007/s1067 6-017-9419-3

Liljamo T, Liimatainen H, Pöllänen M (2018) Attitudes and concerns on automated vehicles. Transport Res Part F Traffic Psychol Behav 59:24-44. https://doi.org/10.1016/j.trf.2018.08.010

Lin P (2016) Why ethics matters for autonomous cars. In: Autonomous Driving, pp 69-85. https://doi.org/10.1007/978-3-662-48847-8_4

Marshall A (2018) Uber's fatal crash and the ethics of testing selfdriving cars/WIRED. https://www.wired.com/story/lose-lose-ethic s-self-driving-public/. Accessed 5 Feb 2019

Maurer M (2016) Introduction. Autonom Driving. https://doi. org/10.1007/978-3-662-48847-8_1

Maurer M, Gerdes JC, Lenz B, Winner H (eds) (2016) Autonomous driving. In: Technical, legal and social aspects. Springer-Verlag, Berlin Heidelberg. https://doi.org/10.1007/978-3-662-48847-8

Miles I (2010) The development of technology foresight: a review. Technol Forecast Soc Chang 77(9):1448-1456. https://doi. org/10.1016/j.techfore.2010.07.016

Molnar LJ, Ryan LH, Pradhan AK, Eby DW, Louis RMS, Zakrajsek JS (2018) Understanding trust and acceptance of automated vehicles: an exploratory simulator study of transfer of control between automated and manual driving. Transport Res Part F Traffic Psychol Behav 58:319-328. https://doi.org/10.1016/j.trf.2018.06.004

Morozov E (2013) The real privacy problem. MIT Technology Review website. https://www.technologyreview.com/s/520426/the-realprivacy-problem/. Accessed 24 Jan 2019 
Nyholm S (2018a) The ethics of crashes with self-driving cars: a roadmap, I. Philos Compass 13(7):e12507. https://doi.org/10.1111/ phc3.12507

Nyholm S (2018b) The ethics of crashes with self-driving cars: a roadmap, II. Philos Compass 13(7):e12506. https://doi.org/10.1111/ phc3.12506

Nyholm S, Smids J (2016) The ethics of accident-algorithms for self-driving cars: an applied trolley problem? Ethic Theory Moral Pract 19(5):1275-1289. https://doi.org/10.1007/s1067 7-016-9745-2

Patel VJ (2017) Think your cellphone uses a lot of data? Report claims autonomous cars will use $4000 \mathrm{~GB}$ in 1 day. FutureCar.com. https ://www.futurecar.com/876/Think-Your-Cellphone-Uses-a-lot-ofData-Report-Claims-Autonomous-Cars-Will-Use-4000-GB-inone-Day. Accessed 5 Feb 2019

Patton MQ (1980) Qualitative evaluation methods. Sage, London

Penmetsa P, Adanu EK, Wood D, Wang T, Jones SL (2019) Perceptions and expectations of autonomous vehicles - a snapshot of vulnerable road user opinion. Technol Forecast Soc Chang 143:9-13. https://doi.org/10.1016/j.techfore.2019.02.010

Peterson R (2012) New technology-old law: autonomous vehicles and California's insurance framework. Santa Clara Law Digital Commons. http://digitalcommons.law.scu.edu/facpubs/337

Ravid O (2014) Don't sue me, I was just lawfully texting and drunk when my autonomous car crashed into you. Southwest Law Rev 44(1):175-207

Ross A (2018) Smart car: Who will win in the battle for the data centre on wheels. Information Age website. https://www.informatio n-age.com/smart-car-data-123473940/. Accessed 5 Feb 2019

Royakkers L, Timmer J, Kool L, van Est R (2018) Societal and ethical issues of digitization. Ethics Inf Technol 20(2):127-142. https:// doi.org/10.1007/s10676-018-9452-x

Ryan M (2019) The future of transportation: ethical, legal, social and economic impacts of self-driving vehicles in the year 2025. Sci Eng Ethics. https://doi.org/10.1007/s11948-019-00130-2

Schoettle B, Sivak M (2014) Public opinion about self-driving vehicles in China, India, Japan, the U.S., the U.K., and Australia. http:// deepblue.lib.umich.edu/bitstream/handle/2027.42/109433/10313 9.pdf? sequence $=1$. Accessed $10 \mathrm{Feb} 2019$

Schwartz P (1998) The art of the long view : planning for the future in an uncertain world. Wiley, New York

Skeete JP (2018) Level 5 autonomy: the new face of disruption in road transport. Technol Forecast Soc Chang 134:22-34. https://doi. org/10.1016/j.techfore.2018.05.003
Stilgoe J, Owen R, Macnaghten P (2013) Developing a framework for responsible innovation. Res Policy 42(9):1568-1580. https://doi. org/10.1016/J.RESPOL.2013.05.008

Taeihagh A, Lim HSM (2018) Governing autonomous vehicles: emerging responses for safety, liability, privacy, cybersecurity, and industry risks. Transport Rev. https://doi.org/10.1080/01441 647.2018.1494640

Templeton G (2018) Autonomous cars data: future cars run on data, not gasolinelGlobalme. Globalme website. https://www.globalme.net/ blog/autonomous-cars-data-not-gasoline. Accessed 5 Feb 2019

Urmson C (2015) How a driverless car sees the road. https://www. youtube.com/watch?v=tiwVMrTLUWg. Retrieved 5 Feb 2019

van Asselt MBA (2010) Foresight in action: developing policy-oriented scenarios. Earthscan, London

Van Der Hoven J (2013) Value sensitive design and responsible innovation. In: Responsible innovation. Wiley, Chichester, UK, pp 75-83. https://doi.org/10.1002/9781118551424.ch4

van Lente H (2012) Navigating foresight in a sea of expectations: lessons from the sociology of expectations. Technol Anal Strat Manag 24:769-782. https://doi.org/10.1080/09537 325.2012.715478

von Schomberg R (2011) Towards responsible research and innovation in the information and communication technologies and security technologies fields. European Commission, Brussels. http:// ec.europa.eu/research/sciencesociety/document_library/pdf_06/ mep-rapport-2011_en.pdf

Wallach W, Allen C (2009) Moral machines. Oxford University Press, Oxford. https://doi.org/10.1093/acprof:oso/9780195374 049.001.0001

Wamsley L (2018) Should self-driving cars have ethics? NPR. NPR website. https://www.npr.org/2018/10/26/660775910/should-selfdriving-cars-have-ethics?t=1549383291947. Accessed 5 Feb 2019

Williamson K, Johanson G (2018) Research methods: information, systems and contexts. Chandos Publishing, UK

Publisher's Note Springer Nature remains neutral with regard to jurisdictional claims in published maps and institutional affiliations. 